\title{
Vie scientifique
}

\section{«Gestion des ressources en eau pour le développement local : gouvernance, institutions et politiques »}

\section{Compte rendu de séminaire (Loskop Dam, Afrique du Sud, 8-11 novembre 2004)}

\author{
Jean-Yves Jamin
}

Agronome, directeur adjoint de l'UMR G-EAU, CIRAD, TA 60/02, 34398 Montpellier cedex 5, France

Ce séminaire, intitulé WRM 2004 (Water Resource Management), a été organisé en Afrique du Sud par le Cirad et l'Université de Pretoria, avec le concours technique et financier du Department of Water Affairs \& Forestry (ministère des Eaux et Forêts), de la Water Research Commission, de l'Association française pour l'étude des irrigations et du drainage, de la Commission internationale des irrigations et du drainage et du Programme coordonné de recherche sur les systèmes irrigués (CemagrefCirad-IRD). Il a réuni environ 200 personnes venues de différents pays d'Afrique australe, d'Afrique de l'Ouest, d'Asie, d'Amérique du Sud, d'Europe et du Pacifique, autour d'une cinquantaine de communications. L'objectif était de faire le point sur les avancées scientifiques dans le domaine de la gestion de l'eau et de les discuter avec différents pays, mais aussi de favoriser un dialogue et des échanges d'expériences entre équipes de recherche, responsables politiques et agents de développement.

À partir du thème général de la gestion des ressources en eau pour un développement durable, l'accent a été mis, dans les présentations, les discussions et les visites de terrain, sur les questions de gouvernance, sur les institutions et sur les politiques de l'eau, tant dans le domaine du développement rural que dans celui du développement urbain ou périurbain, avec une attention particulière pour les interactions entre différents secteurs (irrigation, eau potable, industrie, mines...). Un jeu de rôle a aussi été utilisé pour mettre les participants en situation d'utilisateurs de l'eau et illustrer l'intérêt

Auteur correspondant : jamin@cirad.fr de ces méthodologies participatives. Les questions suivantes ont été abordées :

- au niveau local : productivité de l'eau ; usages multiples de l'eau et conflits d'usage ; économie de la petite irrigation; transfert de la gestion des périmètres irrigués ; usages individuels et usages collectifs ; gouvernance locale de l'eau; droits communautaires sur l'eau et droits commerciaux;

- au niveau régional : allocation des ressources; participation, négociations, conflits ; marché de l'eau entre secteurs d'activité; agences de bassin; interactions villes-campagnes; eau bleue et eau verte; eau pour les pauvres.

Dans tous les pays représentés, la perception des ressources en eau et leurs modes de gouvernance sont en évolution rapide depuis une vingtaine d'années, même si la mise en œuvre des intentions politiques affichées est très inégale. Les points communs sont la prise en compte des questions environnementales, la répartition raisonnée des ressources entre secteurs et la décentralisation des décisions, avec la difficile articulation entre agences techniques de gestion et institutions politiques territoriales. Dans la plupart des cas, les évolutions résultent à la fois d'une pression politique nationale et internationale, de la tension sur les ressources et d'une diversification des usages qui imposent la mise en place de règles de gestion ad hoc.

Dans le cas des pays en développement, l'agriculture garde une place importante dans les préoccupations des décideurs et dans la répartition des ressources, compte tenu de l'importance des questions liées à la sécurité alimentaire et à la lutte contre la pauvreté. 
Les questions pratiques qui se posent sont les mêmes partout : comment répartir l'eau à différentes échelles (locale et régionale) et comment articuler ces échelles? Comment faciliter les négociations entre usagers? Comment éviter ou résoudre les conflits ? Quelles formes d'organisation et quelles institutions mettre en place, et à quelle échelle? Comment combiner les impératifs économiques, sociaux et environnementaux? Quelle place garder pour les utilisateurs les plus pauvres et les plus nombreux, les agriculteurs?

En Afrique du Sud, la législation a été modifiée en 1998, mais son application, malgré un volontarisme politique marqué, soulève des problèmes pratiques, aussi bien en zone rurale qu'en zone urbaine. Les négociations entre secteurs se déroulent dans un contexte déséquilibré - les différents intervenants ayant des poids économiques très variables - et les avancées pratiques sont assez lentes.

Les communications exposées lors de ce séminaire ont parfois eu du mal à dégager les outils facilitant l'émergence institutionnelle et l'apprentissage des acteurs sur les aspects de gestion de l'eau pour le développement. Les présentations d'expériences d'intervention par des équipes associant développeurs, chercheurs et décideurs s'apparentaient souvent à des études de cas et ne dépassaient pas toujours le stade du diagnostic. Ces équipes se sont révélées réticentes, en particulier au niveau des chercheurs, à s'engager dans l'action collective avec les acteurs et dans l'expérimentation d'outils et d'approches constructives.

La session spécifiquement dédiée aux jeux de rôle a été très intéressante. Il s'agit en effet d'outils d'aide à la négociation et à l'apprentissage collectif de la gouvernance. Les expériences présentées reflètent la diversité d'approches et d'attendus. Il apparaît que l'engagement dans l'action et la participation, aussi réussi soit-il au plan social et participatif, ne peut suffire à la démarche scientifique. Le jeu de rôle Aware a été joué au cours de l'atelier, ce qui a permis de mettre les participants en situation de défendre les intérêts des différents utilisateurs de l'eau en Afrique du Sud, dans un contexte de négociations annuelles pour le partage de la ressource sous l'égide des agences de bassin locales. Les différentes expériences de jeux de rôle soulignent bien l'intérêt de ces approches pour accompagner à la fois recherche et apprentissage sur la gouvernance de la ressource, pour soulever de nouvelles questions, pour accompagner des démarches de modélisation plus formelles et quantifiées, pour amplifier le raisonnement des acteurs, favoriser l'exercice de leurs capacités cognitives. Toutefois, des avancées conceptuelles et méthodologiques semblent encore nécessaires. Ces approches posent en fait des problèmes d'apprentissage et de changement de posture à tous les niveaux : chercheurs (facilitateurs-concepteurs), acteurs locaux (joueurs, avec ou sans recul par rapport au jeu), décideurs (devant accepter de nouvelles formes de négociation, de relations et de gouvernance).

Plusieurs modèles ont également été présentés et discutés au cours de l'atelier. Leur intérêt et leurs limites ont ainsi pu être débattus. Leur capacité à intégrer la notion de "risque », fondamentale en agriculture, est ainsi apparue essentielle, ainsi que celle à dépasser la seule dimension économique (parfois économétrique) pour prendre en compte les questions sociales, organisationnelles, politiques... Mais les débats ont surtout porté sur leur aptitude à être utilisés non plus comme simples outils de recherche, mais comme supports effectifs pour l'action avec des agriculteurs individuels ou des communautés locales, pour l'information et la formation avec des étudiants ou des acteurs, pour la négociation entre différentes parties prenantes, ou pour aider à élaborer des outils d'aide à la décision. Si l'intention initiale va en général dans ce sens, les démarches ne sont pas toujours abouties, en particulier avec les petits paysans pauvres ou les communautés rurales des pays en développement.

Les formes d'organisation expérimentées ou mises en place pour une gestion durable de l'eau, prenant en compte l'intérêt des différents secteurs et l'intérêt général des pays concernés, ont été au centre des discussions de plusieurs sessions. Sous différentes formes, les associations d'utilisateurs de l'eau sont aujourd'hui très en vogue, car vues comme une alternative aux États, jugés défaillants. Cependant, les transferts de compétence à ces associations relèvent souvent plus de transferts verticaux, imposés par le haut (bailleurs de fonds en particulier), que de processus réellement participatifs. Des questions importantes restent à préciser, comme la représentation et l'apport respectif des différents utilisateurs de l'eau à ces instances, les relations entre elles et les autres formes d'organisations politiques et territoriales compétentes dans le champ de la gestion de ressources communes, l'étendue des rôles et des décisions qui peuvent leur être dévolus, leur nature commerciale ou de service public, etc. L'équilibre entre secteur public et secteur privé et la nécessité d'établir des passerelles entre différents niveaux de gestion - national, régional, local, individuel - ont également été débattus.

Bien qu'à l'origine de nombreux travaux présentés, il y ait souvent eu des problématiques plutôt agricoles, le séminaire a réussi à intégrer suffisamment de représentants d'autres secteurs d'activité pour que le caractère multi-usage et multisectoriel de l'eau soit bien abordé. Le contexte sud-africain, où les mines sont un gros utilisateur d'eau, où l'eau potable a été l'enjeu de nombreuses luttes durant l'apartheid - avec des séquelles toujours actuelles - et où les préoccupations environnementales sont anciennes (parcs nationaux) mais politiquement non neutres, était particulièrement favorable à cet élargissement des débats. 
La diversité des origines des participants a parfois nécessité des explications un peu longues - mais toujours intéressantes - des contextes particuliers des différents pays. Elle a cependant eu le gros avantage d'obliger chacun à «sortir » de son étude de cas pour s'interroger sur son domaine de validité et en tirer des enseignements plus génériques.

Outre le CD-Rom distribué au cours des travaux, toutes les contributions ont été mises en ligne à l'issue du séminaire (http://wrm2004.cirad.fr). Une sélection d'une vingtaine d'entre elles a été effectuée par l'Université de Pretoria et le Cirad. Un ouvrage collectif les regroupant vient d'être publié : Water Governance for Sustainable Development. Approaches and Lessons from Developing and Transitional Countries. Sylvain Perret, Stefano Farolfi, Rashid Hassan (Eds), Cirad, Earthscan, Cemagref, Ifremer, Inra. 2006, 296 pp.

To access this journal online: www.edpsciences.org 